\title{
Study of Serum Midkine Level in Patients with Rheumatoid Arthritis
}

\author{
Aalaa M. Sweilam, Soheir Abdel Haleem", \\ Abeer Shahba $^{2}$ and Muhammad Tarek Abdel Ghafar ${ }^{1 *}$ \\ ${ }^{1}$ Departments of Clinical pathology, ${ }^{2}$ Department of Internal Medicine, \\ Faculty of Medicine, Tanta University, Egypt \\ *Corresponding author
}

\section{Keywords}

Rheumatoid arthritis, Midkine; DAS-28 score, Activity

\section{Article Info}

Accepted:

12 October 2020 Available Online: 10 November 2020

\begin{abstract}
A B S T R A C T
Rheumatoid arthritis (RA) is a systemic autoimmune disease of unknown etiology. Midkine (MK) is a pleiotropic growth factor prominently expressed during embryogenesis but down-regulated to negligible levels in healthy adults. MK is involved in cell proliferation, differentiation, migration, and survival. The present study aimed to investigate the role of serum midkine level in rheumatoid arthritis (RA) patients and to analyze the relationship between the serum MK level and disease activity. Blood samples were collected from 60 patients with rheumatoid arthritis and twenty healthy individuals served as control group. Serum level of Midkine was measured by Enzyme Linked Immunosorbent Assay (ELISA). Other markers including Erythrocyte sedimentation rate (ESR), C-reactive protein (CRP), rheumatoid factor (RF), and Anti-CCP were estimated. Serum Midkine level was significantly higher in active RA patients than in inactive RA patients and healthy controls. It correlated positively with DAS-28 score in RA patients. ROC curve analysis revealed that the diagnostic efficacy of midkine was of 0.843 and the prediction power of RA activity was of 0.825 . MK was significantly increased in serum of RA patients, and its level was correlated with several clinical markers of RA and DAS-28 score. So, the serum MK level could be a marker of RA disease activity.
\end{abstract}

\section{Introduction}

Rheumatoid arthritis (RA) is characterized by chronic inflammation of multiple joints. The synovial tissue of RA patients features proliferation of synoviocytes, accumulation of inflammatory cells (including lymphocytes and macrophages), production of inflammatory mediators, and angiogenesis (1). Midkine (MK) is a pleiotropic growth factor prominently expressed during embryogenesis but down-regulated to negligible levels in healthy adults. MK is involved in cell proliferation, differentiation, migration, and survival (2).

Many published studies have demonstrated striking MK overexpression compared with healthy controls in various pathologies, including ischemia, inflammation, autoimmunity and, most notably, in many cancers (3). MK expression is detectable in biopsies of diseased, but not healthy, tissues. Significantly, because it is a soluble cytokine, 
elevated MK is readily apparent in the blood and other body fluids such as urine and cerebrospinal fluid (CSF), making MK a relatively convenient, accessible, noninvasive and inexpensive biomarker for population screening and early disease detection (4).

MK expression plays a significant role in inflammatory and autoimmune diseases, including in disease models of rheumatoid arthritis (RA) (5)and multiple sclerosis (MS) (6).Limited human data show that MK is elevated in the synovial fluid and sera of RA patients. Further basic studies investigating MK elevation in human subjects are required to determine whether MK offers utility as a biomarker in RA (4). This study aimed to investigate the role of serum midkine level in rheumatoid arthritis (RA) patients and to analyze the relationship between the serum MK level and disease activity.

\section{Materials and Methods}

The present study was conducted on 60 patients with rheumatoid arthritis (Group 1) and twenty healthy individuals served as control group (Group 2). Patients were selected from outpatient clinic and inpatients of Rheumatology unit, internal medicine department, Tanta University hospitals during the period from January 2018 to August 2018. This study was conducted in clinical pathology department, Tanta University Hospital. They were diagnosed according to the classification criteria of the American College of Rheumatology/ European League Against Rheumatism (7). They were further subdivided according to the disease activity based on Disease Activity Score 28 (DAS-28) into: Group 1A: Thirty patients with active rheumatoid arthritis. They were 6 males and 24 females with age ranged from 20-61 years. Group 1B: Thirty patients with inactive rheumatoid arthritis. They were 4 males and
26 females with age ranged from 23-64 years. The healthy controls were 3 males and 17 females with age ranged from 22-62 years. They were age and sex matched with RA patients. The study was approved by the ethical committee of faculty of medicine, Tanta University and written informed consents were obtained from all participants. Patients with malignant diseases, cardiac diseases, inflammatory diseases and auto immune diseases other than rheumatoid arthritis were excluded from this study.

All patients and controls were subjected to the following: Complete clinical examination, routine laboratory investigations including complete blood cell count (CBC),erythrocyte sedimentation rate (ESR) by Westergren tube method, C- reactive protein (CRP), and rheumatoid factor (RF) via immunoturbidimetric assay on automated chemistry analyzer; Konelab 60 I, Thermo Scientific, Vantaa, Finland, Anti- cyclic citrullinate peptide antibodies (anti-CCP) by electro-chemiluminescence immunoassay (ECLIA) on automated immunoassay analyzer, Cobas e411, Roche Diagnostics $\mathrm{GmbH}$, Mannheim, Germany. The level of disease activity was assessed by DAS-28 and can be interpreted as inactive RA (DAS$28<2.6$ ), and active RA disease (DAS-28 > 2.6)(8).

\section{Immunoassay of serum MidKine level}

Three ml blood were collected from RA patients and controls in a plain tube then centrifuged for 15 minutes at 3000 r.p.m for separation of serum, and then stored at $-20 \mathrm{C}$ until assay of midkine level. The midkine level was estimated via enzyme linked immunosorbent assay (ELISA) (SUNRED., CHINA). The colorimetric detection was performed on a micro plate reader at $450 \mathrm{~nm}$. The standard curve was drawn by plotting the mean absorbance obtained from each standard 
against its concentration with absorbance value on the vertical (Y) axis and concentration on the horizontal (X) axis using linear graph paper. The concentration of the samples was determined directly from this standard curve.

\section{Statistical analysis}

The data collected were tabulated and analyzed by SPSS (statistical package for social science) version 22.0 on IBM compatible computer (SAS Institute, 2005).Chi-square test $(\chi 2)$ was used to study association between two qualitative variables. ANOVA (f) test was used for comparison between three or more groups having quantitative variables. Kruskal-Wallis test was used for comparison between three or more groups not normally distributed having quantitative variables. The ROC (receiver operating characteristic) curves analysis was used to assess the diagnostic performance of midkine and the sensitivity and specificity were calculated. Youden's index was used to determine the optimal cut-off. P- value less than 0.5 is considered significant.

\section{Results and Discussion}

\section{Demographic Characteristics of the studied groups}

In this study, $60 \mathrm{RA}$ patients were recruited and further divided into two groups; group1 A (active RA) and group 1B (inactive RA), in addition to 20 healthy controls as group 2 . No significant difference between the three groups regarding age and sex (Table 1).

\section{Clinical and Laboratory Characteristics of the studied groups}

The inflammatory markers such as ESR and $\mathrm{CRP}$ as well as autoimmune markers as RF and Anti-CCP levels were significantly higher in active RA patients than inactive RA patients and healthy controls. Moreover, serum Midkine level was significantly higher in active RA patients than inactive RA patients and healthy controls. As expected the DAS-28 score was significantly higher in active RA patients than inactive RA patients (Table 1).

Correlation between serum Midkine level and demographic, clinical and laboratory characteristics of the patients' groups

Pearson correlation analysis between serum midkine level with age, ESR, CRP, RF, AntiCCP levels, and DAS-28 score in RA patients revealed that serum midkine level with ESR, $\mathrm{RF}$, serum Anti-CCP, DAS-28 score levels in active RA patients and with ESR level and DAS-28 score in inactive RA patients (Table 2).

Performance characteristics of serum MidKine in the studied group

Roc curve analysis of the performance characteristics of the MidKine was performed. For RA diagnosis, Midkine had AUC of 0.843 whereas, serum anti-CCP had AUC of 0.833 , and RF had AUC of 1.000 (Figure 1). At a cutoff value of $>230 \mathrm{pg} / \mathrm{mL}$, midkine was of $91.0 \%$ sensitivity and $65.0 \%$ specificity in differentiating RA patients from healthy subjects. For prediction of active RA patients, midkine had AUC of 0.825 whereas, ESR had AUC of 0.959, and CRP had AUC of 0.984 (Figure 2). At a cutoff value of $>310$ $\mathrm{pg} / \mathrm{mL}$, midkine was of $83.0 \%$ sensitivity and $70.0 \%$ specificity in differentiating active from inactive RA patients.

Rheumatoid arthritis (RA) is a chronic inflammatory disease that affect from $0.5 \%$ to $1 \%$ of population, therefore it considered the most common chronic inflammatory autoimmune disease. Early diagnosis for RA 
is very important and it is difficult because early presentation of RA patients may confuse with other inflammatory arthritis. Moreover, the classical clinical picture of RA tends to present after the progression of the disease. Additionally, treatment with antiinflammatory drugs or corticosteroids may mask symptoms and signs (9).This study was designed to investigate the role of serum midkine level in rheumatoid arthritis (RA) patients and to analyze the relationship between the serum MK level and disease activity.

Table.1 Demographic, clinical, and laboratory characteristics of the studied groups

\begin{tabular}{|c|c|c|c|c|}
\hline & $\begin{array}{c}\text { Group 1A } \\
(\mathbf{n}-30)\end{array}$ & $\begin{array}{c}\text { Group 1B } \\
(\mathbf{n}=\mathbf{3 0})\end{array}$ & $\begin{array}{c}\text { Group2 } \\
(n=20)\end{array}$ & P-value \\
\hline Age (years) & $43.27 \pm 10.03$ & $41.93 \pm 10.39$ & $44.2 \pm 9.89$ & 0.729 \\
\hline Sex (Male/Female) n & $6 / 24$ & $4 / 26$ & $3 / 17$ & 0.771 \\
\hline $\operatorname{ESR}(\mathbf{m m})$ & $81.27 \pm 17.78^{\mathrm{ab}}$ & $14.87 \pm 6.20$ & $7.95 \pm 2.48$ & $<0.001 *$ \\
\hline CRP (mg/l) & $48.87 \pm 26.49^{\mathrm{ab}}$ & $5.07 \pm 2.88$ & $3.95 \pm 1.47$ & $<0.001 *$ \\
\hline RF (IU/ml) & $99.47 \pm 22.21^{\mathrm{ab}}$ & $27.13 \pm 16.27^{\mathrm{a}}$ & $3.50 \pm 1.61$ & $<0.001 *$ \\
\hline Anti-CCP (U/ml) & $90.67 \pm 46.09^{\mathrm{ab}}$ & $45.17 \pm 24.68$ & $26.15 \pm 24.65$ & $<0.001 *$ \\
\hline DAS score & $4.72 \pm 1.06$ & $1.79 \pm 0.52$ & ---- & $<0.001 *$ \\
\hline SerumMidkine (pg/ml) & $458.33 \pm 157.92^{\mathrm{ab}}$ & $301.67 \pm 73.02$ & $236.5 \pm 63.10$ & $<0.001 *$ \\
\hline
\end{tabular}

Table.2 Correlation of serum Midkine level with age, ESR, CRP, RF, Anti-CCP levels and DAS score in RA patients

\begin{tabular}{|c|c|c|c|c|}
\hline \multicolumn{5}{|c|}{ Midkine (pg/ml) } \\
\hline & \multicolumn{2}{|c|}{ Active RA } & \multicolumn{2}{|c|}{ Inactive RA } \\
\hline & $\mathbf{r}$ & P-value & $\mathbf{R}$ & P-value \\
\hline Age (Years) & 0.014 & 0.533 & 0.001 & 0.853 \\
\hline $\operatorname{ESR}(\mathrm{mm} / \mathbf{1 h})$ & 0.371 & $0.001 *$ & 0.253 & $0.005^{*}$ \\
\hline CRP (mg/dl) & 0.048 & 0.246 & 0.012 & 0.578 \\
\hline RF (IU/ml) & 0.430 & $0.001 *$ & 0.017 & 0.486 \\
\hline Anti-CCP (U/ml) & 0.251 & $0.005^{*}$ & 0.098 & 0.092 \\
\hline DAS score & 0.289 & $0.002 *$ & 0.178 & $0.020 *$ \\
\hline
\end{tabular}

Fig.1 Roc curve analysis of the MidKine, Anti-CCP, RF levels between RA patients and healthy controls

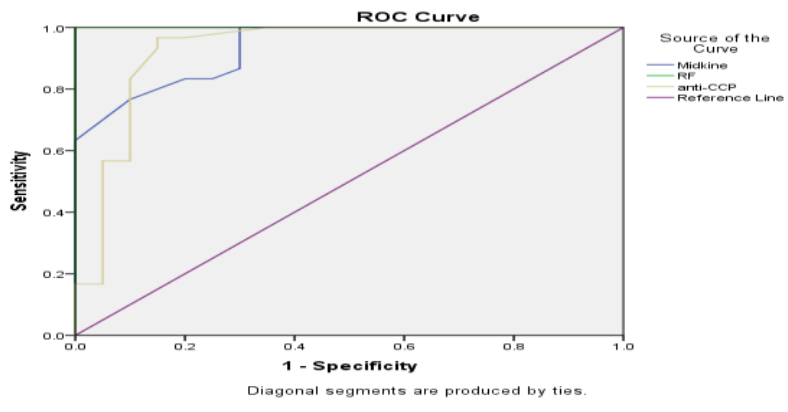


Fig.2 Roc curve analysis of the MidKine, CRP, ESR levels between active RA patients and inactive RA patients

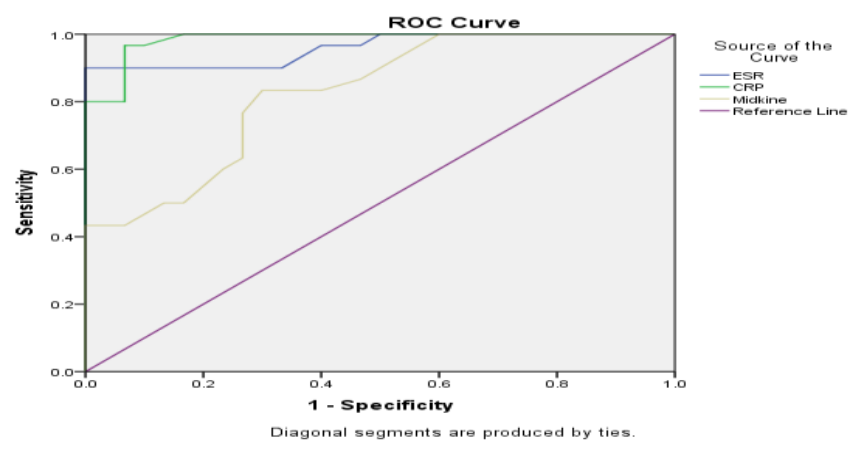

ESR and CRP are considered as markers of inflammation which indicate the disease activity in RA patients. In the present study, ESR and CRP were high in active RA patients group compared to healthy controls group and inactive RA. Consistent with our results, study conducted by Ranganath et al., (10)reported that ESR is one of laboratory indicators of the disease activity in RA patients. Wislowska and Jablonska, (11) and Massoud et al., (12) found that CRP was elevated in patients with early RA.

In this study, serum RF and Anti-CCP levels were significantly higher in RA patients than healthy subjects. RF is the most sensitive laboratory serologic marker for the diagnosis of RA according to the American College of Rheumatology (ACR). However, the specificity of RF is relatively low because there is a $50 \%$ positive rate of $\mathrm{RF}$ in patients with other connective-tissue diseases, such as systemic lupus erythematosus (SLE), primary Sjögren syndrome, and dermatomyositis, with some infections; and in healthy elderly persons, which limits its diagnostic value (13). Anti-CCP antibodies have the power to predict the development of RA in patients with early and undifferentiated arthritis (14). The new criteria for diagnosis of RA have been introduced and anti-CCP, together with RF, is included therein (7).
As regard serum midkine level, the results of the present work showed that there was a significant elevation of serum midkine levels in active RA patients compared to inactive RA patients and healthy subjects. For RA diagnosis, midkine had AUC of 0.843. At a cutoff value of $>230 \mathrm{pg} / \mathrm{mL}$, midkine was of $91.0 \%$ sensitivity and $65.0 \%$ specificity in differentiating RA patients from healthy subjects. In agreement of the results of the present work, Shindo et al., (1) showed that the serum MK level is elevated in RA patients and is correlated with clinical indexes of RA. Previous study has shown that the serum MK level is elevated in RA patients and is correlated with the RF titer (5).

In this study, serum midkine level correlated positively with DAS-28 score which indicates that the serum MK level could be a useful marker of RA disease activity. For prediction of active RA patients, Midkine had AUC of 0.825 . At a cutoff value of $>310 \mathrm{pg} / \mathrm{mL}$, midkine was of $83.0 \%$ sensitivity and $70.0 \%$ specificity in differentiating active from inactive RA patients. Shindo et al., (1)found that the serum MK level was correlated with DAS28-ESR and that MK tended to be decreased after treatment with an anti-TNF-a antibody.

MK has been implicated in a wide number of biological functions including e.g. 
angiogenesis under hypoxic conditions (15), cell survival (16), and tumor growth(17). Multiple studies also suggested an important role for MK in several pathological inflammatory conditions (18). Accordingly, MK is significantly up regulated in inflamed synovial tissue during an active rheumatoid arthritis flare in contrast to synovial tissue of healthy patients (19).

MK was expressed in the RA synovial lining and synovial fluid, with minimal MK expression in OA synovium and synovial fluid (5). This is further confirmed by findings in a mouse arthritis model, where leukocyte infiltration and joint damage are markedly attenuated in MK-/- mice (5).Thus, MK could be a potential therapeutic target in patients with RA.

Stimulation with MK enhanced the production of IL-6, IL-8, and CCL2 by cultured RSFs. IL-8 is a chemokine (C-X-C motif) ligand 8 (CXCL8), which induces blood vessel formation and angiogenesis and also exhibits a chemoattractant activity for neutrophils and dendritic cells (20). CCL2 is thought to induce migration of monocytes into inflamed RA synovial tissue (21). Moreover, it was reported that MK induces neutrophil migration (19), so MK could both directly and indirectly promote inflammatory cell accumulation in RA synovial tissue and synovial fluid. It has been reported that MK enhances endothelial cell proliferation and vascular growth (22) and also up-regulates the differentiation of osteoclasts in vitro (5). Therefore, MK may contribute to angiogenesis and bone destruction in the synovial tissues in RA, as well as stimulating RSFs.

In conclusion, MK was significantly increased in serum of RA patients, and its level was correlated with several clinical markers of RA and DAS-28 score. So, the serum MK level could be a marker of RA disease activity. However, large number of cases and follow up the patients over time is recommended to determine prognostic value of serum midkine level in RA patients. Also, it is recommended to study serum midkine level in comparison with synovial midkine level and its correlation with disease severity.

\section{Disclosure}

The authors report no conflicts of interest.

\section{Funding}

This research did not receive any fund.

\section{Author Contributions}

All authors contributed equally in the writing of this manuscript.

\section{References}

1.Shindo E, Nanki T, Kusunoki N, Shikano $\mathrm{K}$, Kawazoe M, Sato H, et al. The growth factor midkine may play a pathophysiological role in rheumatoid arthritis. Modern rheumatology. 2017; 27: 54-9.

2.Weckbach LT, Muramatsu T, Walzog B. Midkine in inflammation. The Scientific World Journal. 2011; 11: 2491-505.

3.Muramatsu T. Midkine, a heparin-binding cytokine with multiple roles in development, repair and diseases. Proceedings of the Japan Academy Series B, Physical and biological sciences. 2010; 86: 410-25.

4.Jones DR. Measuring midkine: the utility of midkine as a biomarker in cancer and other diseases. British journal of pharmacology. 2014; 171: 2925-39.

5.Maruyama $\mathrm{K}$, Muramatsu $\mathrm{H}$, Ishiguro $\mathrm{N}$, Muramatsu T. Midkine, a heparin- 
binding growth factor, is fundamentally involved in the pathogenesis of rheumatoid arthritis. Arthritis and rheumatism. 2004; 50: 1420-9.

6.Sonobe $\mathrm{Y}$, Li $\mathrm{H}$, Jin $\mathrm{S}$, Kishida $\mathrm{S}$, Kadomatsu K, Takeuchi $\mathrm{H}$, et al. Midkine inhibits inducible regulatory $\mathrm{T}$ cell differentiation by suppressing the development of tolerogenic dendritic cells. Journal of immunology Baltimore, Md: 1950. 2012; 188: 2602-11.

7.Aletaha D, Neogi T, Silman AJ, Funovits J, Felson DT, Bingham CO, 3rd, et al. 2010 Rheumatoid arthritis classification criteria: an American College of Rheumatology/European League Against Rheumatism collaborative initiative. Arthritis and rheumatism. 2010; 62: 2569-81.

8.Fransen J, Stucki G, van Riel PLCM. Rheumatoid arthritis measures: Disease Activity Score DAS., Disease Activity Score-28 DAS28., Rapid Assessment of Disease Activity in Rheumatology RADAR., and Rheumatoid Arthritis Disease Activity Index RADAI. 2003; 49: S214-S24.

9.Aref M.I, H. A. Cartilage Oligomeric Matrix Protein as New Marker in Diagnosis of Rheumatoid Arthritis. Mod Chem Appl., 2015:151.

10.Ranganath VK, Khanna D, Paulus HE. ACR remission criteria and response criteria. Clinical and experimental rheumatology. 2006; 24: S-14-21.

11.Wisłowska M, Jabłońska B. Serum cartilage oligomeric matrix protein COMP. in rheumatoid arthritis and knee osteoarthritis. Clinical rheumatology. 2005; 24: 278-84.

12.Saghafi M, Khodashahi M, Saadati N, Azarian A, Rezaieyazdi Z, Salehi M, et al. Relationship between cartilage oligomeric matrix protein COMP. and rheumatoid arthritis severity. Electronic physician. 2017; 9: 5940-7. 13.Shen R, Ren X, Jing R, Shen X, Chen J, Ju $\mathrm{S}$, et al. Rheumatoid Factor, AntiCyclic Citrullinated Peptide Antibody, C-Reactive Protein, and Erythrocyte Sedimentation Rate for the Clinical Diagnosis of Rheumatoid Arthritis. Laboratory medicine. 2015; 46: 226-9.

14.Bizzaro N, Bartoloni E, Morozzi G, Manganelli S, Riccieri V, Sabatini P, et al. Anti-cyclic citrullinated peptide antibody titer predicts time to rheumatoid arthritis onset in patients with undifferentiated arthritis: results from a 2-year prospective study. Arthritis research \& therapy. 2013; 15:R16.

15. Weckbach LT, Groesser L, Borgolte J, Pagel JI, Pogoda F, Schymeinsky J, et al. Midkine acts as proangiogenic cytokine in hypoxia-induced angiogenesis. American journal of physiology Heart and circulatory physiology. 2012; 303: H429-38.

16. Cohen S, Shoshana OY, Zelman-Toister E, Maharshak N, Binsky-Ehrenreich I, Gordin M, et al., The cytokine midkine and its receptor RPTP $\zeta$ regulate $B$ cell survival in a pathway induced by CD74. Journal of immunology Baltimore, Md: 1950. 2012; 188: 259-69.

17.Güngör $\mathrm{C}$, Zander $\mathrm{H}$, Effenberger $\mathrm{KE}$, Vashist YK, Kalinina T, Izbicki JR, et al. Notch signaling activated by replication stress-induced expression of midkine drives epithelialmesenchymal transition and chemoresistance in pancreatic cancer. Cancer research. 2011; 71: 5009-19.

18.Wang J, Takeuchi H, Sonobe Y, Jin S, Mizuno T, Miyakawa S, et al. Inhibition of midkine alleviates experimental autoimmune encephalomyelitis through the 
expansion of regulatory $\mathrm{T}$ cell population. Proceedings of the National Academy of Sciences of the United States of America. 2008; 105: 3915-20.

19.Takada T, Toriyama K, Muramatsu H, Song XJ, Torii S, Muramatsu T. Midkine, a retinoic acid-inducible heparin-binding cytokine in inflammatory responses: chemotactic activity to neutrophils and association with inflammatory synovitis. Journal of biochemistry. 1997; 122: 453-8.

20.Ritchlin C. Fibroblast biology. Effector signals released by the synovial fibroblast in arthritis. Arthritis research. 2000; 2: 356-60.

21.Hayashida K, Nanki T, Girschick H, Yavuz S, Ochi T, Lipsky PE. Synovial stromal cells from rheumatoid arthritis patients attract monocytes by producing MCP-1 and IL-8. Arthritis research. 2001; 3: 118-26.

22 Choudhuri R, Zhang HT, Donnini S, Ziche $\mathrm{M}$, Bicknell R. An angiogenic role for the neurokines midkine and pleiotrophin in tumorigenesis. Cancer research. 1997; 57: 1814-9.

\section{How to cite this article:}

Aalaa M. Sweilam, Soheir Abdel Haleem, Abeer Shahba and Muhammad Tarek Abdel Ghafar. 2020. Study of Serum Midkine Level in Patients with Rheumatoid Arthritis. Int.J.Curr.Microbiol.App.Sci. 9(11): 1408-1415. doi: https://doi.org/10.20546/ijcmas.2020.911.165 